\title{
Latest nuclear emulsion technology
}

\section{Production, readout and interaction analysis}

Hiroki Rokujo a, Hiroaki Kawahara, Ryosuke Komatani, Misaki Morishita, Toshiyuki Nakano, Naoto Otsuka, and Masahiro Yoshimoto

Nagoya University, Furo-cho, Chikusa-ku, Nagoya 464-8602, Japan

\begin{abstract}
Nuclear emulsion is a extremely high-resolution 3D tracking detector. Since the discovery of the pion by C.F. Powell et al. in 1946, experiments with nuclear emulsions have contributed to the development of particle physics. (e.g. the OPERA collaboration reported the discovery of $v_{\mu} \rightarrow v_{\tau}$ oscillations in appearance mode in 2015) The technology of nuclear emulsion still keeps making progress. Since 2010, we have introduced a system of nuclear emulsion gel production to our laboratory in Nagoya University, and have started self-development of the new gel, instead of from the photographic film companies. Moreover, a faster automated emulsion scanning system is developed. Its scanning speed reaches $4000 \mathrm{~cm}^{2} / \mathrm{h}$, and the load for analyzing becomes more and more lighter. In this presentation, we report the status of nuclear emulsion technologies for cosmic ray experiments.
\end{abstract}

\section{Introduction}

Nuclear emulsion is an ultra high-precision positionsensitive detector. It consists of mostly gelatin and crystals of silver bromide $(\mathrm{AgBr})$. The crystals penetrated by a charged particle grow up to silver grains (the diameter is about $0.8 \mu \mathrm{m}$ ) via a chemical development process, then we can observe the trajectory of the particle as a line of black points using optical microscopes. The intrinsic position accuracy is expected to be $200 / \sqrt{12}=56 \mathrm{~nm}$. In addition, although typical electronic trackers consist of readout channels in two or more independent projections for $3 \mathrm{D}$ reconstruction, nuclear emulsion directly gives $3 \mathrm{D}$ position information of the trajectory.

S. Kinoshita discovered that the radiation of alpha decay is a "track" of a charged particle by the observation of nuclear emulsion in 1915 [1]. C. F. Powell and his colleagues discovered the decay chain of $\pi \rightarrow \mu+v$ in nuclear emulsions exposed to cosmic rays [2].

Double-side poured nuclear emulsion plate realizes a precise measurement of incident angle. In the 1950's, a Japanese group developed a chamber, called Emulsion Cloud Chamber (ECC), that had a sandwich structure of nuclear emulsion and other material plates (carbon, lead, etc.). ECCs mounted on balloons or airplanes enabled to measure angles of jet particles from high-energy cosmicray interactions. A fire ball model of multiple meson production in nucleon-nucleon collisions [3] and the discovery of charmed meson in cosmic-ray interactions [4] were produced by the observation of ECCs in the era when no accelerator was in Japan.

Though the observation and the measurement of tracks in nuclear emulsion had been done by human eyes and microscopes, the realization of fully automated scanning

a e-mail: rokujo@flab.phys.nagoya-u.ac.jp systems enabled high resolution and large-scale experiments which use the advantages of nuclear emulsion. For example, the OPERA experiment discovered $v_{\mu} \rightarrow$ $v_{\tau}$ oscillation in appearance mode on the basis of these technologies [5].

Here, we report the recent improvements of the nuclear emulsion technology since 2010.

\section{Emulsion gel production}

In 2010 we introduced a system of emulsion gel production to the laboratory in Nagoya University, and started to supply the new gel instead of relying on photographic film companies. Figure 1 shows a nuclear emulsion gel produced in Nagoya on the left, and an image of $\mathrm{AgBr}$ crystals captured by SEM after removing gelatin on the right.

Our self-production system enables us to develop ambitious nuclear emulsion gels. We succeeded in the development of a high-sensitive nuclear emulsion by increasing the occupancy of $\mathrm{AgBr}$ crystals from typically $30-35 \%$ to $55 \%$. Figure 2 shows a comparison of microscopic images between two type of nuclear emulsion. The grain density (the average number per unit length) of the silver particles of this new type of gel is two times higher than that of a typical gel.

The size control of $\mathrm{AgBr}$ crystals is also studied. $200 \mathrm{~nm}$-diameter crystal has been used for detection of minimum ionization particles for a long time. Recently, we produced various-sized crystals according to the purposes of experiments. The minimum-sized crystals (the diameter is $30-40 \mathrm{~nm}$ ) are being developed for dark matter directional searches [6], and the maximum-sized crystals $(\sim 800 \mathrm{~nm})$ for the long term observation requesting the stability of the latent image [7].

(c) The Authors, published by EDP Sciences. This is an Open Access article distributed under the terms of the Creative Commons Attribution License 4.0 (http://creativecommons.org/licenses/by/4.0/). 

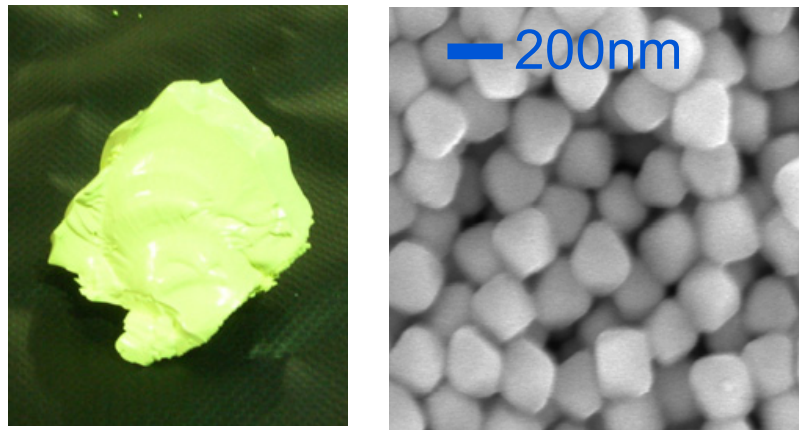

Figure 1. Nuclear emulsion gel produced in Nagoya on the right, and SEM image of $\mathrm{AgBr}$ crystals after removing gelatin on the left.

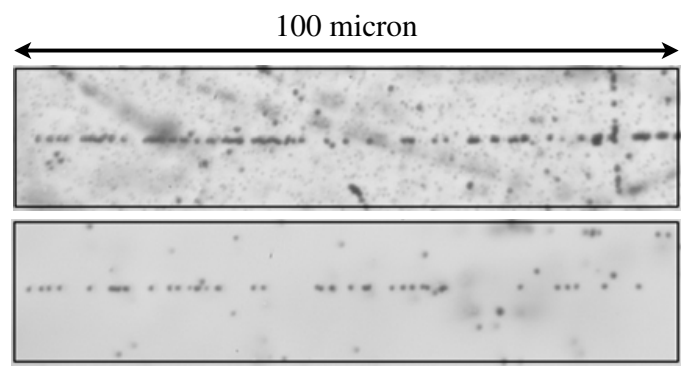

Figure 2. Microscopic view of highly sensitive film (upper) and typical film (lower).

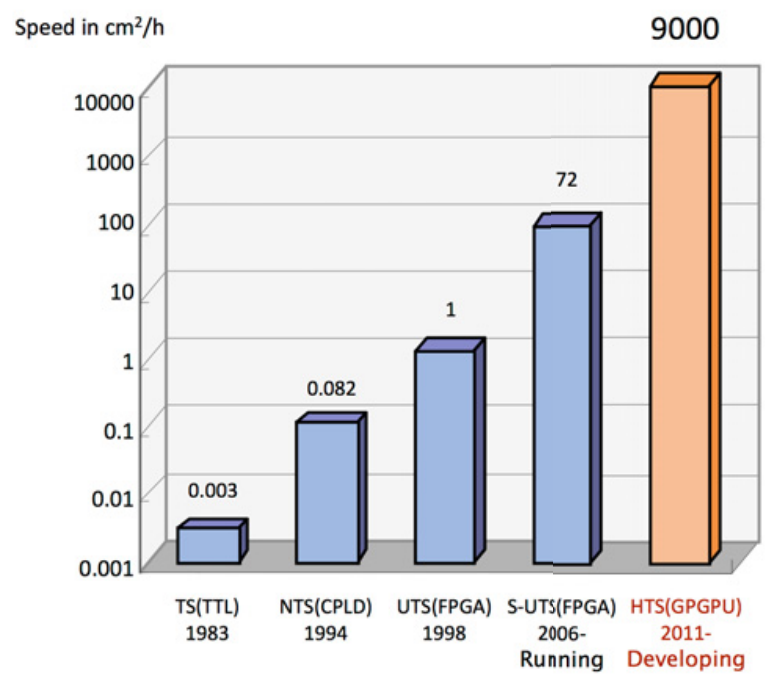

Figure 3. Evaluation of scanning speed by generations of Track Selector.

The environment of the mass production of films is constructed in the laboratory. We have already produced the nuclear emulsion film at a speed of $100 \mathrm{~m}^{2} /$ year and supplied several experiments. In a few years, the production speed will increase to about $1000 \mathrm{~m}^{2} /$ year for accelerator neutrino experiments, balloon-borne experiments, muon radiography projects, etc.

\section{Emulsion readout system}

The concept of fully automated emulsion readout system, Track Selector (TS), was invented by K. Niwa [8], and was

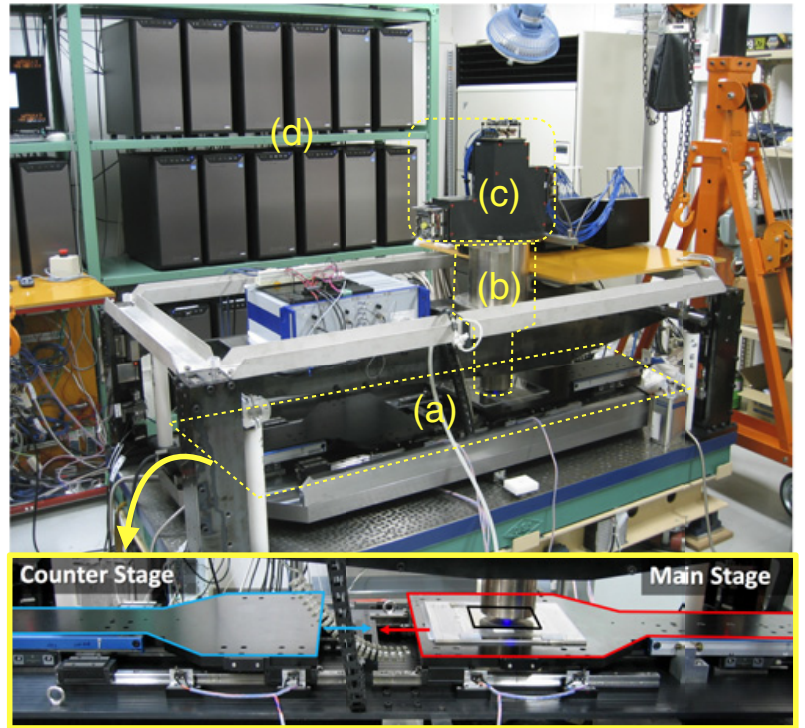

Figure 4. Picture of HTS. It consists of (a) precision stages which move to the next view precisely and quickly after capturing, (b) a huge objective lens that realizes a $5 \mathrm{~mm} \times 5 \mathrm{~mm}$-size FOV, (c) 72 mosaic CMOS sensors that cover the large FOV (each 2M pixels, $340 \mathrm{fps}$.), and (d) $36 \mathrm{PSs}$ for realtime process of track-recognizing with $27 \mathrm{~GB} / \mathrm{s}$ image data.

put to practical use in the 1990's. The latest TS is called Hyper Track Selector (HTS). HTS has been developed in Nagoya University since 2011. Figure 3 shows scanning speed by generations of TS, and HTS is designed for $0.9 \mathrm{~m}^{2} /$ hour.

HTS consists of precision stages, a huge objective lens, 72 mosaic CMOS sensors, and 36 PSs. These are shown in Fig. 4. The most characteristic point of HTS is a very wide field of view (FOV), $5 \mathrm{~mm} \times 5 \mathrm{~mm}$. The FOV of HTS is 600 times larger than that of the previous system, S-UTS. To cover its FOV with $0.4 \mu \mathrm{m}$ pitch pixel, the image is divided into six imaging planes through prisms, and $12 \mathrm{CMOS}$ sensors (each $2 \mathrm{M}$ pixels, $340 \mathrm{fps}$ ) capture it at each plane. The image data from 72 sensors are sent to $36 \mathrm{PCs}$, then the track recognition and the calculation (angles, positions, etc.) are processed immediately. A motor-driven XY axes stage moves to the next view precisely and quickly. At that time, the counter stage moves in the opposite direction from the main stage to cancel acceleration.

Figure 5 shows the pulse height distribution obtained by the high-sensitive film and HTS. TS recognizes a track from 16 images (32 images in a double-side poured film) captured over changing a focal plane, and the number of hit layers in a signal track is called the pulse height. By improvement of the grain density, clear signals were observed. Figure 6 shows the trackfinding efficiency obtained by the high-sensitive film and HTS. At each incident angle from $\tan \theta=0.0$ (a vertical angle) to $\tan \theta=2.0$, more than $95 \%$ was evaluated [9].

The operation of HTS was started in 2015 at half of the designed speed. Nuclear emulsion films employed in the GRAINE-2015 balloon experiment were scanned as the first practical data taking of HTS, and it took three months to finish $41 \mathrm{~m}^{2}$ in total [10]. 
Table 1. Candidates of previous emulsion experiments for the archive project.

\begin{tabular}{llc}
\hline Experiments & Original purpose & Total Area for Scanning \\
\hline RUNJOB (balloon experiment) & primary cosmic-ray observation & $120 \mathrm{~m}^{2}$ \\
JACEE (balloon experiment) & primary cosmic-ray observation & $900 \mathrm{~m}^{2}$ \\
CHORUS (accelerator $v$ experiment) & search for $v_{\mu} \rightarrow v_{\tau}$ oscillations & $400 \mathrm{~m}^{2}$ \\
DONUT (accelerator $v$ experiment) & detection of $v_{\tau}$ & $100 \mathrm{~m}^{2}$ \\
\hline
\end{tabular}

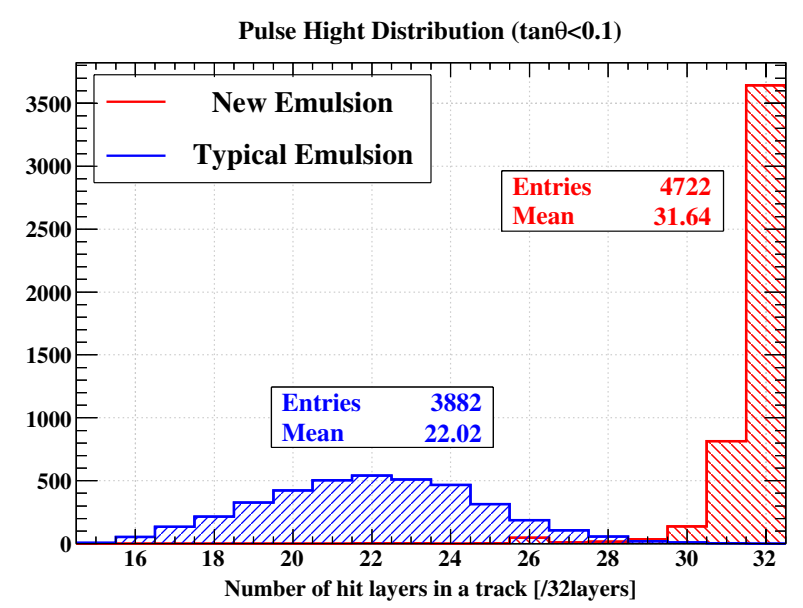

Figure 5. Pulse height distributions. Red histogram was given by high-sensitive emulsion film and HTS. Blue histogram was given by typical emulsion film (OPERA type).

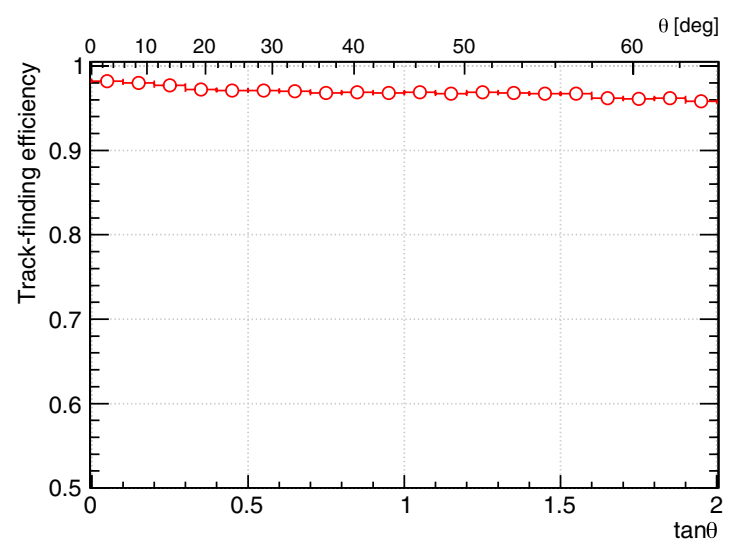

Figure 6. Track-finding efficiency as a function of incident angle obtained by the high-sensitive film and HTS.

\section{Applications for cosmic-ray experiments}

\subsection{Balloon-borne gamma-ray telescope}

GRAINE is a project aimed at a precise observation of gamma-ray sources (the energy region is sub-GeV/GeV) with a balloon-borne nuclear emulsion chamber. The gamma-ray telescope with the original timestamp technique $[11,12]$ has the following advantages: high angular resolution ( $0.1 \mathrm{deg}$ at $1 \mathrm{GeV})$; polarization sensitive; large aperture area $\left(>10 \mathrm{~m}^{2}\right)$. Recently, a small-scale observation was made to demonstrate the performance of the detector [13].

\subsection{Emulsion archives project}

We also plan to archive "digital" data of emulsion plates of past experiments and to make it public. In previous

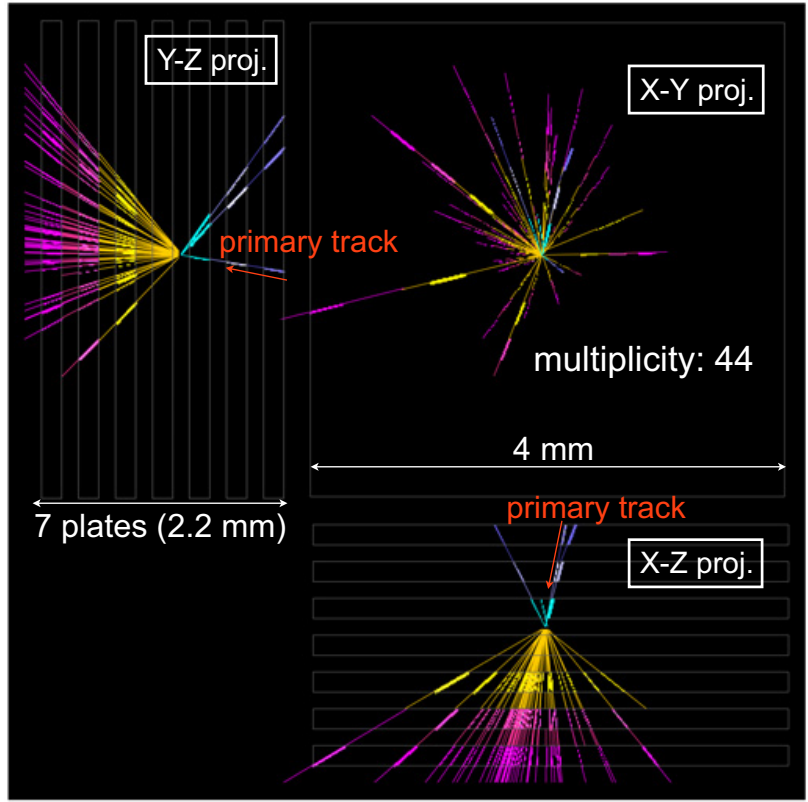

Figure 7. Cosmic-ray interaction event recorded in a balloonborne chamber. It was detected by an offline analysis method under development.

(accelerator and cosmic-ray) emulsion experiments, the analysis was performed by focusing on special interesting events. A re-scan data set of the whole of the detector volume provides for minimum-bias and no-dead-time analysis without an event trigger of another detector (electronic counters or X-ray films). We will start a test scan of old emulsion plates (see Table 1) and discuss about a standard data format etc, toward opening archive data to the public.

\subsection{Study for cosmic ray interactions}

We are developing an offline analysis method to detect hadronic interactions recorded in the emulsion chamber. Figure 7 shows a sample event recorded in a balloon-borne chamber. If a systematic analysis flow is established, it is possible to search for short-lived particles from a large number of cosmic-ray interactions, and to measure the production rate of charmed particles at balloon altitudes. In accelerator experiments, the measurement of differential cross section of $\mathrm{D}_{s}$ mesons produced in proton interactions is starting at the CERN SPS. Nuclear emulsions can record all tracks emitted from the vertex with $4-\pi$ angular acceptance, and cover the wide range of rapidity in the laboratory frame. A precise comparison of the topological values between hadron interaction models will also be tried. 


\section{Conclusion}

We report the status of nuclear emulsion technologies for cosmic ray experiments. The highest quality emulsion films became available by introducing self-gel production system to the laboratory level. The emulsion readout systems are getting increasingly faster and the next generation system is being planned. These innovations of nuclear emulsion technology will lead cosmic ray physics to the next stage.

This work was supported by JSPS KAKENHI Grant Number 26247039, 26105510, and 16K17691.

\section{References}

[1] S. Kinoshita and H. Ikeuti, Philosophical Magazine and Jounal of Science Ser. 6, 29, 171, pp. 420-425 (1915)

[2] C.M.G. Lattes, G.P.S. Occhialini and C.F. Powell, Nature 160, 453 (1947)
[3] K. Niu et al., Nuovo cimento 10, p. 994, (1958)

[4] K. Niu et al., Progress of theoretical physics 46(5), 1644-1646 (1971)

[5] N. Agafonova et al. Physical review letters 115(12), $121802(2015)$

[6] T. Naka et al., Nuclear Instruments and Methods A 718, 519-521 (2013)

[7] T. Ariga et al., Journal of Instrumentation 11(03), P03003 (2016)

[8] K. Niwa et al., Proc. Int. Cosmic Ray Symp. High Energy Phenomena, Tokyo, p. 149 (1974)

[9] K. Ozaki et al., Journal of Instrumentation 10(12), P12018 (2015)

[10] H. Rokujo et al., Proc. of ISVHECRI 2016, Moscow (2016)

[11] S. Takahashi et al., Nuclear Instruments and Methods A 620, 192 (2010)

[12] H. Rokujo et al., Nuclear Instruments and Methods A 701, 127 (2013)

[13] S. Takahashi et al., Prog. Theor. Exp. Phys. 2015, 043H01 (2015) 